\title{
PARALLELE WELTEN. INWIEFERN LITERATURWISSENSCHAFT UND SPRACHWISSENSCHAFT VONEINANDER PROFITIEREN KÖNNEN
}

\author{
URSULA BOCK \\ Europa-Universität Viadrina - Frankfurt (Oder)
}

\begin{abstract}
The essay starts with a discussion about language as the central medium of human communication. Language is the object of research within linguistics, that analyse its structure as well as its pragmatic and communicative character. But also literature and research in literature reflect about language, its prospects and also its limits of mutual understanding. Problems of communication often arise in intercultural environments, especially under the restraints of emigration or foreign rules.

Nowadays problems increase because of tendencies of globalization, that require the learning of foreign languages, but also the acquaintance with foreign cultures. Under these circumstances foreign language teaching gets more and more important to procure intercultural communicative skills. Whereas the chances of literature appear to be rather limited on this field.
\end{abstract}

Im Vorwort einer Veröffentlichung der Kulturwissenschaftlichen Fakultät der Viadrina „Was sind Kulturwissenschaften?" finden sich folgende Überlegungen zur spezifischen Ausrichtung der eigenen Arbeit:

Wir betrachten die ,Kulturwissenschaft" nicht als eine einheitliche neue Disziplin, die verbindlich definiert werden könnte, sondern als eine offene Verflechtung von Wissenschaften, die sich zusammengefunden haben, um neue Phänomene der Kultur zu untersuchen, die mit den alten Disziplingrenzen nur schwer zu erfassen wären. (Kittsteiner 2004:8)

Es geht also darum, den zunächst eigenen disziplinären Blickwinkel zu öffnen für einen weiteren interdisziplinären Diskurs über die Fachgrenzen hinaus. Daß diese Öffnung theoretisch oder programmatisch nicht vorgegeben war, läßt die Entstehungsgeschichte dieser wie auch anderer Kulturwissenschaftlicher Fakultäten erkennen: Demnach wurden die Lehrkörper „von Wissenschaftspolitikern und akademischen Gründungsgremien (...) zusammenberufen (...) und müssen nun nachträglich darauf reflektieren, wer und was sie sind.“ (Kittsteiner 2004:9). Diese Anmerkung verdeutlicht, daß den Kern der Fakultäten Lehrstühle und Professuren bilden, die zunächst nach den herkömmlichen Disziplinen ausgerichtet und 
mit entsprechenden Fachvertretern besetzt sind. Der interdisziplinäre Ansatz entsteht damit im Austausch von Wissenschaftlern verschiedener Disziplinen, die sich fächerübergreifend über gemeinsame Projekte verständigen und nicht aus der Arbeit innerhalb eines Lehrstuhles. So stellt die Arbeit einer Literaturwissenschaftlerin an einem sprachwissenschaftlichen Lehrstuhl auch heute noch eine Besonderheit und eine besondere, gleichwohl aber auch sehr reizvolle Herausforderung dar. Das will ich an dieser Stelle vor dem Hintergrund meiner Erfahrungen am Lehrstuhl für Fremdsprachendidaktik der Viadrina erläutern. Meine beispielhaften Überlegungen betreffen das Thema Sprache aus dem Blickwinkel der Linguistik wie auch der Literatur und Literaturwissenschaft und zielen darauf ab, für einen interdisziplinären Dialog auch innerhalb eines Faches zu werben.

\section{Sprache und Kommunikation}

Schon Humboldt verficht 1820 in seinem Vortrag „Über das vergleichende Sprachstudium in Beziehung auf die verschiedenen Epochen der Sprachentwicklung“ die These: „Der Mensch ist nur Mensch durch Sprache; um aber die Sprache zu erfinden müsste er schon Mensch seyn.“(Humboldt 1994:20). Diese kurze Feststellung hat von ihrer Aktualität in den vergangenen fast zweihundert Jahren nichts eingebüßt. Sie weist auf die nach wie vor ungelösten Fragen von Sprachentstehung und -entwicklung hin, die in der Sprachwissenschaft ebenso wie in der Psychologie, der Literaturwissenschaft und der Philosophie diskutiert werden. Unstrittig ist: Die Sprache stellt das wesentliche Mittel der menschlichen Kommunikation dar. Sie bestimmt die zwischenmenschlichen Beziehungen und spiegelt ihre Entwicklung wider. Allerdings untersuchen Wissenschaftler die Bedingungen ihrer Entstehung wie auch ihrer Funktion aus jeweils unterschiedlicher Perspektive. Die Sprachwissenschaft verfolgt im Rahmen dieser Diskussion einen zumeist positivistischen Ansatz: Sie sucht nach den Regularien, die eine Sprache beschreiben und erfassen können. Darüber hinaus hat sie Kommunikationsbedingungen und -situationen im Blick mit dem Ziel, die Voraussetzungen zu formulieren, unter denen Kommunikation erfolgreich verläuft bzw. unter denen gestörte Kommunikation wieder in die richtigen Bahnen gelenkt werden kann.

Als Gegenstand der Reflexion ist Sprache in der Dichtung ebenso wie in der Literaturwissenschaft seit jeher ein zentrales Thema. Die Schriftstellerin Ulla Hahn hat in ihrem Roman Das verborgene Wort (2001) dem Zauber und der Faszination von Sprache nachgespürt. Die Begegnung des Mädchens Hildegard mit der Welt der Wörter und Buchstaben wird als emphatische Erfahrung beschrieben, die ihr Leben entscheidend verändert:

Jedes, aber auch jedes Wort der Welt könne man lesen, lockte uns der Lehrer, wenn man nur diese sechsundzwanzig Buchstaben, Alphabet genannt, kenne. Sogar neue 
Worte könne man erfinden. Aber damit hätten wir noch viel Zeit. Zuerst einmal sollten wir die Buchstaben lernen. A, sagte der Lehrer und malte etwas auf die Tafel: A, wiederholte er. Buchstaben kamen aus den Tönen. (Hahn 2001:55)

Der schöpferische Umgang mit Sprache und später mit Literatur eröffnet dem Kind einen Fluchtweg aus den engen Verhältnissen ihres rheinischen Elternhauses hin zu einer Parallelwelt nicht gekannter großer Gefühle, fremder Schicksale, phantastischer Geschichten.

Während der Sprachwissenschaftler Laute nüchtern nach der Art oder dem Ort ihrer Artikulation, zum Beispiel nach der Öffnung des Mundes oder Stellung der Zunge vorne und hinten, oben und unten definiert, malt Ulla Hahn sie kunstvoll in leuchtenden Farben und fesselnden Bildern aus:

Ein langes a war ein Staunen, ein kurzes beinah ein Nein. $\mathrm{O}$ freute und wunderte sich. I war Ekel und Abscheu. Mit langen Us kam das Gruseln. (Hahn 2001:6)

Häufiger allerdings artikulieren Schriftsteller ihre Vorbehalte gegenüber dem Gelingen kommunikativen Handelns, so Hugo von Hofmannsthal zu Beginn des 20. Jahrhunderts in dem berühmten „Chandos-Brief" (1902). Darin berichtet der - fiktive - Lord Chandos über das Erlebnis einer Sprachkrise, das sich zu einer Bewußtseins- und Identitätskrise ausweitet. ,...die abstrakten Worte, deren sich doch die Zunge naturgemäß bedienen muß, um irgendwelches Urteil an den Tag zu geben, zerfielen mir im Munde wie modrige Pilze“ (Hofmannsthal 1991:48f.), lautet seine Selbstdiagnose, die aufzufassen ist als Krise des Individuums, das seine Umwelt und sich selbst gedanklich nicht mehr zu erfassen und sprachlich nicht mehr zu bewältigen vermag.

Der Sprachphilosoph Ludwig Wittgenstein versucht demgegenüber in seinen Philosophischen Untersuchungen (1953), das Problem von Verstehen und Benennen dadurch zu lösen, daß er das Sprachspiel einführt: Sprecher handeln kommunikativ, indem sie übereinkommen, sich auf gemeinsame Bezeichnungen zu einigen, sie wenden Sprachspiele an: Der Lehrer zeigt auf einen Gegenstand und benennt ihn, seine Schüler übernehmen den Namen (Wittgenstein 1971:16). Da sich das Verfahren auch auf abstrakte Begriffe übertragen läßt, sorgt das Sprachspiel dafür, daß Verständigung möglich wird.

Der Dichter Peter Handke hat (wie andere auch) die Grenzen des Sprachspiels vielfach thematisiert: Wenn die Gewißheit versagt, daß Dinge mit einem Begriff zu entschlüsseln sind, wenn die Sprecher an der Sprache und ihrem Verstehen zu zweifeln beginnen, dann öffnet sich ein Abgrund der Existenzangst. Es geht ihnen wie dem Boten, der über den zugefrorenen Bodensee reitet und tot zusammenbricht, als er erfährt, welchen Weg er unbewußt zurückgelegt hat (so in dem Stück Der Ritt über den Bodensee 1970). Wenn Sprache nicht mehr selbstverständlich ist, stürzt der Mensch in eine Lebenskrise „,...dann erstarren 
alle wieder und ziehen sich zusammen, machen sich ganz klein, wie erfrierend" (Handke 1970:102), dann droht selbst das Wort „Stecknadel“, einen Nervenzusammenbruch auszulösen (Handke 1970:55).

\title{
2. Fremde und eigene Sprache
}

Wenn bereits die Muttersprache nicht mehr voraussetzungslos verstanden und benutzt werden kann, um wieviel mehr muß der Sprecher einer Fremdsprache - vor allem wenn er dazu gezwungen wird - Entfremdung und Identitätsverlust spüren. Die Gründe können vielfältig sein: So berichtet Inge Deutschkron von den Schwierigkeiten jüdischer Emigranten, die während der nationalsozialistischen Diktatur aus Deutschland nach England geflohen sind, in dem neuen Land und in der neuen Sprache heimisch zu werden:

\begin{abstract}
Deutsch war nun einmal die Sprache, in der die Emigranten ihre Gedanken und ihre Gefühle am treffendsten auszudrücken in der Lage waren. Es muß ihnen sehr bald bewußt geworden sein, daß die neue, zu erlernende Sprache weder ein Ersatz für die Muttersprache werden, noch jemals in Qualität, Genauigkeit und Differenziertheit der von Geburt gelernten Sprache gleichen würde. Das betraf den Wortschatz wie den Akzent. (Deutschkron 2001:27f.)
\end{abstract}

Der Philosoph und Literaturtheoretiker Jacques Derrida, als jüdischer Araber in Algerien geboren, erlebt mit der Besetzung seines Landes durch die Franzosen den Zwang, sich eine fremde Sprache aneignen zu müssen. Er wird damit nicht nur politisch, sondern auch kulturell kolonialisiert.

Nur weil die Sprache nicht sein natürliches Eigentum ist, kann er (der Beherrscher) sie historisch durch die Gewalt kultureller Usurpation, die wesenhaft kolonial ist, als seine auferlegen. Weil es kein natürliches, eigentliches Eigentum der Sprache gibt, ist die Gewalt, die sie veranlaßt, allein die der Aneignung. So daß jeder sagen kann: ich habe nur eine Sprache und das ist nicht meine. Meine eigene, meine eigentliche Sprache ist mir eine Fremdsprache. Meine Sprache, die einzige die ich spreche, ist die Sprache des Anderen. (Derrida 1997:22)

Folgerichtig beobachtet der Literaturwissenschaftler Anselm Haverkamp eine Überlagerung vieler Sprachen durch koloniale Spuren:

Die postkolonialen Gemeinsprachen, sofern sie nicht wie die lateinamerikanischen Sprachen zu neuen regional gültigen Nationalsprachen geworden sind, sondern als Weltsprachen überregional gesprochen werden, tragen ihre koloniale Vergangenheit gleichwohl in sich. (Haverkamp 1997:9) 
Tatsächlich findet heute eine umfassende Auseinandersetzung um Verstehen, Übertragen und Lernen fremder Sprachen statt, also um die Frage der interkulturellen Kommunikation. Die Bewahrung kultureller Identität gilt als wichtigstes Ziel bei der Vermittlung von Fremdsprachen wie auch der Übersetzung fremdsprachiger Literatur. Wir leben in einer globalisierten Welt, in der sich Ströme von Menschen - freiwillig oder gezwungen - zwischen unterschiedlichen Kulturkreisen bewegen, in der Handel und Märkte, Wissenschaft und Kunst international ausgerichtet sind. Vor allem junge Leute fühlen sich nicht mehr an ihre nationalen Grenzen gebunden: als Reisende, Austauschschüler und -studenten oder Arbeitnehmer lernen sie andere Länder und damit andere kulturelle Hintergründe kennen. Das hat Einfluß auf den Umgang miteinander wie auch auf den Fremdsprachenunterricht.

Als Teil des Gesamtunterrichts zählt auch der Fremdsprachenunterricht zu einem Ort, an dem interkulturelles Lernen möglich und nötig ist. Das bedeutet, dass nicht allein fremdsprachliche Kommunikation eingeübt wird, sondern es vielmehr um die Vermittlung des kulturellen Hintergrundes geht, um ein Wechselspiel des „Fremdund Eigenverstehens", heißt es bei Waldemar Pfeiffer. (Pfeiffer 2002:132)

Der bekannte Übersetzer polnischer Literatur, Karl Dedecius, sieht seine Aufgabe darin, als Übersetzer Brücken zwischen Kulturen zu bauen. Er glaubt an die Möglichkeit, Fremdheit der Kultur durch Sprache und Sprachverstehen zu überwinden.

Lernen wir zusammenleben. Die Sprachen sind dazu da, diesen höheren Zweck zu erfüllen. Indem wir Sätze übersetzen, Sinn übertragen, Anmut und Harmonie aus einem Mutterboden in einen anderen verpflanzen. (Dedecius 1993:489)

Natürlich ist Dedecius in bezug auf die Nachbarn Polen und Deutschland vorbehaltlos zuzustimmen, denn beide Länder haben ihre Wurzeln in der abendländischen Tradition und sind seit Jahrhunderten kulturell eng verbunden. Diffiziler wird die Betrachtung, wenn die Übersetzung Texte aus Ländern unterschiedlicher Kulturen betrifft. Indirekt deutet der Ursprung des Begriffs ,übersetzen" das Problem bereits an: er leitet sich vom lateinischen ,interpretare" her. Gleichzeitig heißt ,interpretieren“ in der litraturwissenschaftlichen Praxis, einen Text zu verstehen und zu deuten. Der Begriff ist also vielschichtig, und die Kunst der Übersetzung umfaßt zwei Bereiche: den der Übertragung und den der Auslegung. Schon Humboldt ist sich sicher, daß die Übersetzung von einer in eine andere Sprache nicht selbstverständlich gelingen kann:

Dagegen giebt es eine weit größere Menge von Begriffen, und auch grammatischen Eigenheiten, die so unlösbar in die Individualität ihrer Sprache verwebt sind, dass sie 
weder am bloßen Faden der inneren Wahrnehmung zwischen allen schwebend erhalten, noch, ohne Umänderung in eine andre übertragen werden können. (Humboldt 1994:25)

Humboldt bezieht seine Äußerungen unmittelbar auf das Studium indianischer Sprachen, die er den europäischen gegenüber stellt. Ähnliche Erfahrungen beschreibt Gyatri Spivak am Beispiel der Übertragung von Literatur aus Indien (aus dem Bengali) in das Englische. Spivak erkennt in der unterschiedlichen geographischen Herkunft von Literatur auch ihren Anteil an kolonialer Vergangenheit sowie an kultureller, sozialer und geschlechtsspezifischer Differenz, und

daß es manchmal nicht ausreicht, nur eine tiefe Bindung an korrekte politischkulturelle Überzeugungen zu haben, die in den Einzelheiten des persönlichen Lebens erfahren werden. Vielmehr muß sowohl die Geschichte der Sprache als auch die Geschichte des Zeitpunktes, zu dem der Autor lebt, sowie die Geschichte der Sprache in der und als Übersetzung im Gewebe Gestalt annehmen. (Spivak 1997:75)

Spivak plädiert deshalb für die Anerkennung kultureller Differenz in der Literatur und in der Übersetzung.

Dieser Unterschiede müssen wir uns bewußt sein. Und wir dürfen den Unterschied zwischen ethnischen Minoritäten in der Ersten Welt und der Bevölkerungsmehrheit in der Dritten nicht vernachlässigen. (Spivak 1997:85)

\section{Interkulturelles (Miß-)Verstehen}

Damit aber wird ein Problem berührt, daß meiner Meinung nach bis heute nicht vollständig gelöst ist. Die kulturelle Differenz ist allein sprachlich nicht in jedem Fall zu überwinden. Ich will das an dem Beispiel des international sehr erfolgreichen, auf Englisch geschriebenen Romans der indischen Schriftstellerin Arundhati Roy Der Gott der kleinen Dinge (1997) verdeutlichen. Das Buch behandelt den Konflikt innerhalb einer indischen Familie, deren Angehörige zum Teil auch in Europa und den USA leben oder gelebt haben. Der nuancierten Verästelung der Geschichte, die Zwischentöne der in Indien angesiedelten Tragödie, die in der Konfrontation zwischen Stadt und Land, zwischen Mann und Frau, zwischen Europa und Asien angelegt ist, vermag der europäische Leser nicht ohne weiteres zu folgen. Allenfalls wird er die Fremdheit und Exotik des Schauplatzes und der Ereignisse interessant finden können. So erscheinen in der deutschen Ausgabe des Romans Begriffe wie Paravans, Pelayas, Pulayas (Roy 1999:91), Inquilab Zindabad (Roy 1999:98) oder bhagan (Roy 1999:104), deren Bedeutung dem mit 
Indien nicht vertrauten Leser unklar bzw. unverständlich bleiben müssen. Mithin besteht die Gefahr, daß Leser Texte anderer Kulturkreise, um hier einen Begriff Huntingtons zu verwenden, nur oberflächlich verstehen, weil die kulturellen Besonderheiten eines Originals nicht oder nur schwer zu übersetzen sind.

Welche Erkenntnis läßt sich aus diesen Feststellungen für den interdisziplinären kulturwissenschaftlichen Ansatz gewinnen? Einmal sicherlich, daß die moderne Fremdsprachenvermittlung, die mit Hilfe von neuen Medien und intensivem persönlichen Austausch Sprache in ihrem kulturellen Umfeld lehrt, den richtigen Ansatz verfolgt. Darüber hinaus wird auch klar, daß sich Sprache und Literatur, Sprachwissenschaft und Literaturwissenschaft sinnvoll ergänzen können. Sprache läßt sich mit Unterstützung von Literatur oft anschaulicher vermitteln, spannender erklären, wozu Ulla Hahn das Beispiel lieferte. Auch lassen sich Erkenntnisse der Sprachwissenschaft, zum Beispiel im Bereich von Semantik und Textanalyse, sinnvoll auf die Erschließung literarischer Texte anwenden. Zu lernen ist aber auch, daß die Erwartungen an das Verstehen von Sprache und die Möglichkeiten der Kommunikation nicht in jedem Fall zu erfüllen sind. Zweifel, inwieweit Verstehen und Verständigung durch Sprache herzustellen sind, formuliert die Literatur. Sie problematisiert darüber hinaus die Schwierigkeit, Gedanken und Gefühle adäquat auszudrücken. Und sie lehrt, daß kulturelle Differenz nicht ohne weiteres zu überbrücken ist. Das hat historische ebenso wie geographische und soziale Gründe. Diese zu erkennen und zu überwinden, gegebenenfalls aber auch zu akzeptieren, darin liegt eine Aufgabe kulturwissenschaftlicher Arbeit. Dabei kann die Literatur hilfreich sein - auch im sprachwissenschaftlichen Unterricht.

\section{LITERATUR}

Dedecius, Karl (1993): Kulturvermittlung oder die Brücken der Sprache. In: Ewa Kobylińska u.a.: Deutsche und Polen. 100 Schlüsselbegriffe. München: Piper Verlag, S. 483-493.

Derrida, Jacques (1997): Die Einsprachigkeit des Anderen oder die Prothese des Ursprungs. In: Anselm Haverkamp (Hg.): Die Sprache der Anderen. Frankfurt am Mai: Fischer Taschenbuch Verlag, S. 15-41.

Deutschkron, Inge (2001): Emigranto. Vom Überleben in fremden Sprachen. Berlin: Transit Verlag. Hahn, Ulla (2001): Das verborgene Wort. Roman. Stuttgart, München: Deutsche Verlags Anstalt.

Handke, Peter (1970): Der Ritt über den Bodensee. Frankfurt am Main: suhrkamp.

Haverkamp, Anselm (1997): Zwischen den Sprachen. In: Ders. (Hg.): Die Sprache der Anderen. Frankfurt am Main: Fischer Taschenbuch Verlag, S. 7-12.

Hofmannsthal, Hugo von (1991): Ein Brief. In: Sämtliche Werke. Bd. XXXI. Hg.. Ellen Ritter. Frankfurt am Main: S. Fischer Verlag, S. 45-55.

Humboldt, Wilhelm von (1994): Über die Sprache. Hg.: Jürgen Trabant. Tübingen, Basel: Francke Verlag.

Kittsteiner, Heinz Dieter (2004) (Hg.): Was sind Kulturwissenschaften? München: Fink Verlag.

Pfeiffer, Waldemar (2002): Möglichkeiten und Grenzen der interkulturellen Sprachvermittlung. In: Glottodidactica Nr. XXVIII (2000) Poznań, S. 125-139. 
Roy, Arundhati (1997): Der Gott der kleinen Dinge. München: Taschenbuch Goldmann.

Spivak, Gayatri (1997): Die Politik der Übersetzung. In: Anselm Haverkamp (Hg.): Die Sprache der Anderen. Frankfurt am Main: Fischer Taschenbuch Verlag, S. 65-93.

Ludwig, Wittgenstein (1971): Philosphische Untersuchungen. Frankfurt am Main: suhrkamp. 